\title{
Tau Accumulation and Defective Autophagy: A Common Pathological Mechanism Underlying Repeat-Expansion-Induced Neurodegenerative Diseases?
}

\author{
Li Song1 ${ }^{1} \cdot$ Luoying Zhang ${ }^{1,2}$ (D)
}

Received: 15 May 2020/ Accepted: 3 June 2020/Published online: 5 November 2020

(C) Shanghai Institutes for Biological Sciences, CAS 2020

Repetitive DNA tracts (microsatellites) occur thousands of times throughout the human genome, and their expansion is known to cause many diseases. Expansion of CAG repeats encoding polyglutamine (polyQ) tracts is a pathological cause of nine neurodegenerative diseases: spinal and bulbar muscular atrophy, Huntington's disease, dentatorubropallidoluysian atrophy, and six autosomal dominant forms of spinocerebellar ataxia (SCA1, 2, 3, 6, 7, and 17) [1]. In 2011, two independent groups found an intronic GGGGCC (G4C2) expansion mutant in C9orf12 as the most common genetic cause of both amyotrophic lateral sclerosis (ALS) and frontotemporal dementia (FTD) [2,3]. Evidence from these studies supports the idea that both loss-of-function and gain-of-function mechanisms contribute to these diseases. Bidirectional transcription of mutant C9orf72 produces sense and antisense hexanucleotide repeat expansions. These RNA tracts can undergo repeat-associated non-AUG translation to produce aggregated dipeptides. The aggregated substances can lead to nuclear dysfunction, affecting RNA splicing and transcription as well as causing DNA damage. Proteostasis pathways have also been implicated in disease pathology, including impairments in autophagy and lysosomal function, the unfolded protein response, and the endoplasmic reticulum [4], as well as the ubiquitin-proteasome system [5]. Besides gain-of-function

Luoying Zhang

zhangluoying@hust.edu.cn

1 Key Laboratory of Molecular Biophysics of the Ministry of Education, College of Life Science and Technology, Huazhong University of Science and Technology, Wuhan 430074, China

2 Institute of Brain Research, Huazhong University of Science and Technology, Wuhan 430074, China mechanisms, the $\mathrm{G} 4 \mathrm{C} 2$ repeats $(\mathrm{G} 4 \mathrm{C} 2 \mathrm{r})$ also interfere with the expression of the C9orf72 gene product, leading to a decreased protein level. Therefore, both C9orf 72 deficiency and toxic gain-of-function mechanisms can lead to various disruptions of nucleotides and proteins, although the pathological mechanisms remain largely unclear (Fig. 1).

Tau, a microtubule-associated protein first discovered in 1975 and encoded by MAPT, functions in microtubule assembly and stabilization (Fig. 2). Aggregation of tau is a defining pathological feature of several neurodegenerative diseases that are collectively known as tauopathies, including Alzheimer's disease, progressive supranuclear palsy, corticobasal degeneration, Huntington's disease, and frontotemporal dementia with parkinsonism-17 [6]. Under pathological conditions, the altered modification of tau can result in detachment of tau from microtubules, leading to defects in microtubule stabilization. Neurofibrillary tangles, which consist of hyper-phosphorylated tau acting in a toxic gain-of-function manner, interfere with the transport of vesicles and other cargos [7].

Previous studies implied a synergistic interaction between $\mathrm{G} 4 \mathrm{C} 2 \mathrm{r}$ expansion and tau that leads to neurodegeneration-related pathological features in both humans and flies. Moreover, the protein and phosphorylation levels of tau are increased by G4C2r expansion [8]. Hyperphosphorylated tau that tends to aggregate into insoluble neurofibrillary tangles are found in tauopathy patients. A new finding published in this issue of Neuroscience Bulletin demonstrates a potential role of tau in the development of G4C2r expansion-induced neurodegenerative diseases [9].

In this study by Wen and colleagues, overexpression of $(G G G G C C)_{30}$ repeats $\left[(G 4 C 2 r)_{30}\right]$ induced ALS-related phenotypes in flies, including neurodegeneration, motor 


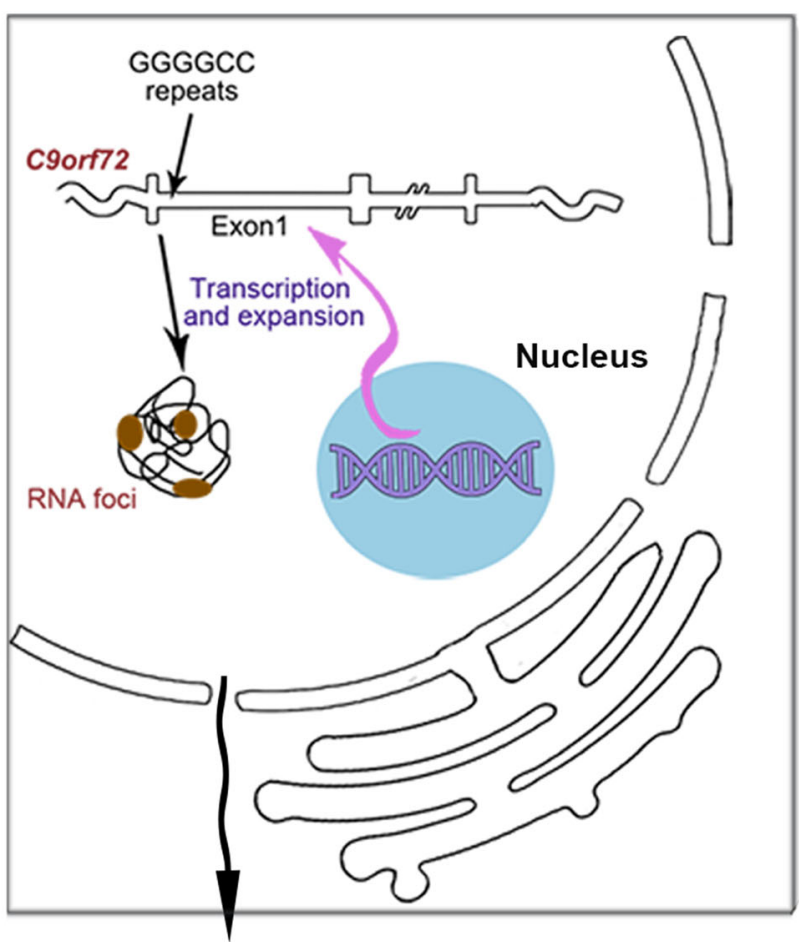

DNA impairment

Mitochondrial dysfunction

Autophagy and lysosomal dysfunction

Impaired ubiquitin proteasome system

Axonnal transport dysfunction

Synapse loss

...

Fig. 1 Cellular processes implicated in FTD and ALS associated with GGGGCC repeat expansion

deficits, and shortened life span, indicating that the fly model recapitulates some of the major symptoms in human ALS patients [9]. Importantly, dtau homozygous (dtau -/-) or heterozygous knockout (dtau +/-) successfully rescued the ALS-related phenotypes in flies overexpressing $(G 4 C 2 r)_{30}$, implying that tau mediates the neurological perturbations caused by $\mathrm{G} 4 \mathrm{C} 2 \mathrm{r}$ expansion [9]. Consistent with the findings at the cellular and behavioral levels, expanded $\mathrm{G} 4 \mathrm{C} 2 \mathrm{r}$ increases the tau protein levels and phosphorylated-tau levels in both fly and human cells, further suggesting a key role of tau in the expanded G4C2r-induced neurodegeneration [9].

In ALS patients with C9ORF72 expansion, cytoplasmic p62/sequestosome-1 (SQSTM1)-positive inclusions have been observed in neurons of the cerebellum, hippocampus, and neocortex. Given that SQSTM1/p62 functions as an autophagy receptor, this suggests autophagy deficits in these patients. Molecular characterizations in cell lines and primary neurons indicate that C9ORF72 regulates the initiation of autophagy by interacting with the Rabla and

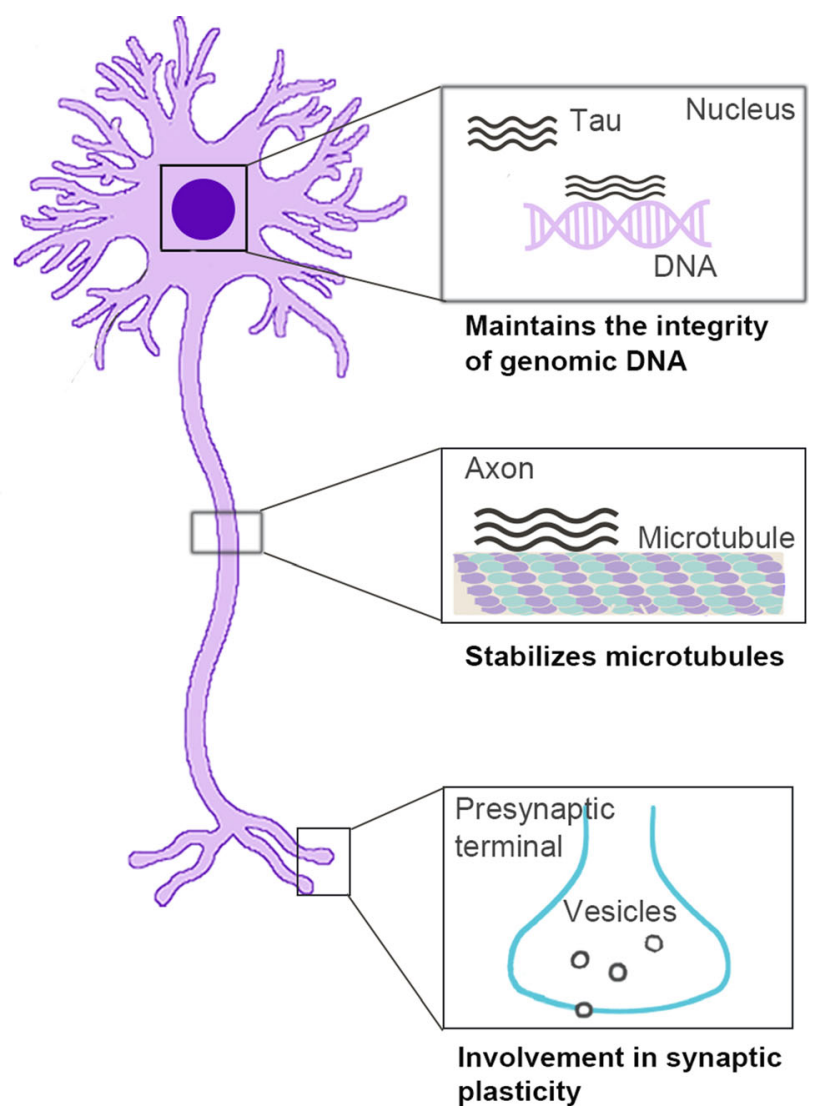

Fig. 2 Potential roles of tau in different subcellular compartments

ULK1 autophagy initiation complex. Depletion of $C 9 \mathrm{orf} 72$ leads to p62/SQSTM1-positive inclusions in these cells [10]. In the current study, on the other hand, the authors found that expansion of G4C2r in both fly and human cells impaired autophagic flux by inhibiting autophagosomeautolysosome fusion, which contributes to tau aggregation [9]. Bcl2-associated athanogene 3 (BAG3) is a member of the BAG family of co-chaperones and interacts with heat shock protein (Hsp) 70. As an autophagy regulator, BAG3 can cooperate with the autophagy receptor SQSTM1/p62 to mediate the clearance of soluble tau and its phosphorylated forms. During this degradation process, BAG3 regulates autophagic flux through interaction with the post-synaptic cytoskeletal protein synaptopodin in mature neurons [11]. In this study, the authors showed that expanded G4C2r expression reduced BAG3/starvin mRNA levels and overexpression of $B A G 3$ rescued the impaired autophagic flux [9]. Taken together, these results reveal a potential pathological mechanism underlying neurological diseases caused by G4C2 expansion: the expanded G4C2r expression decreases $B A G 3$ expression, leading to reduced autophagic flux and tau accumulation. Given that tau also appears to mediate HTT-induced neurodegeneration, alterations in the BAG3-autophagy-tau pathway may contribute to the pathological processes in Huntington's disease and 
perhaps other repeat-expansion-induced neurodegenerative diseases as well. Further explorations are needed to validate this, as well as to determine whether these findings in flies hold true in mammals. Nonetheless, the current work offers new hope for possible therapeutic targets for these devastating neurodegenerative diseases.

\section{References}

1. Lieberman AP, Shakkottai VG, Albin RL. Polyglutamine repeats in neurodegenerative diseases. Annu Rev Pathol 2019, 14: 1-27.

2. DeJesus-Hernandez M, Mackenzie IR, Boeve BF, Boxer AL, Baker M, Rutherford NJ, et al. Expanded GGGGCC hexanucleotide repeat in noncoding region of C9ORF72 causes chromosome 9p-linked FTD and ALS. Neuron 2011, 72: 245-256.

3. Renton AE ME, Waite A, Simon-Sanchez J, Rollinson S, Gibbs $\mathrm{JR}$, et al. A hexanucleotide repeat expansion in C9ORF72 is the cause of chromosome 9p21-linked ALS-FTD. Neuron 2011: 245-256.

4. Wang R, Xu X, Hao Z, Zhang S, Wu D, Sun H, et al. Poly-PR in C9ORF72-related amyotrophic lateral sclerosis/frontotemporal dementia causes neurotoxicity by clathrin-dependent endocytosis. Neurosci Bull 2019, 35: 889-900.

5. Haeusler AR, Donnelly CJ, Rothstein JD. The expanding biology of the C9orf72 nucleotide repeat expansion in neurodegenerative disease. Nat Rev Neurosci 2016, 17: 383-395.

6. Wang Y, Mandelkow E. Tau in physiology and pathology. Nat Rev Neurosci 2016, 17: 5-21.

7. Ballatore C, Lee VM, Trojanowski JQ. Tau-mediated neurodegeneration in Alzheimer's disease and related disorders. Nat Rev Neurosci 2007, 8: 663-672.

8. He H, Huang W, Wang R, Lin Y, Guo Y, Deng J, et al. Amyotrophic lateral sclerosis-associated GGGGCC repeat expansion promotes Tau phosphorylation and toxicity. Neurobiol Dis 2019, 130: 104493.

9. Wen X, An P, Li H, Zhou Z, Sun Y, Wang J, et al. Tau accumulation via reduced autophagy mediates GGGGCC repeat expansion-induced neurodegeneration in Drosophila ALS models. Neurosci Bull 2020. https://doi.org/10.1007/s12264-02000518-2.

10. Webster CP, Smith EF, Grierson AJ, De Vos KJ. C9orf72 plays a central role in Rab GTPase-dependent regulation of autophagy. Small GTPases 2018, 9: 399-408.

11. Ji C, Tang M, Zeidler C, Hohfeld J, Johnson GV. BAG3 and SYNPO (synaptopodin) facilitate phospho-MAPT/Tau degradation via autophagy in neuronal processes. Autophagy 2019, 15: $1199-1213$. 